\title{
BMJ Open Evaluation of the quality of cardiopulmonary resuscitation according to vehicle driving pattern, using a virtual reality ambulance driving system: a prospective, cross- over, randomised study
}

Jin Ho Beom, ${ }^{1}$ Min Joung Kim, ${ }^{1}$ Je Sung You, ${ }^{1}$ Hye Sun Lee, ${ }^{2}$ Ji Hoon Kim, ${ }^{1}$ Yoo Seok Park, ${ }^{1}$ Dong Min Shin, ${ }^{3}$ Hyun Soo Chung ${ }^{1}$

To cite: Beom JH, Kim MJ, You JS, et al. Evaluation of the quality of cardiopulmonary resuscitation according to vehicle driving pattern, using a virtual reality ambulance driving system: a prospective, cross-over, randomised study. BMJ Open 2018;8:e023784. doi:10.1136/ bmjopen-2018-023784

- Prepublication history for this paper is available online. To view these files, please visit the journal online (http://dx.doi. org/10.1136/bmjopen-2018023784).

Received 28 April 2018 Revised 16 July 2018 Accepted 22 August 2018

(A) Check for updates

(c) Author(s) (or their employer(s)) 2018. Re-use permitted under CC BY-NC. No commercial re-use. See rights and permissions. Published by BMJ.

${ }^{1}$ Department of Emergency Medicine, Yonsei University College of Medicine, Seoul, Republic of Korea

${ }^{2}$ Biostatistics Collaboration Unit, Yonsei University College of Medicine, Seoul, Republic of Korea

${ }^{3}$ Department of Paramedic Science, Korea National University of Transportation, Jeongpyeong, Republic of Korea

Correspondence to

Dr Yoo Seok Park;

pys0905@yuhs.ac

\section{ABSTRACT}

Objectives To analyse changes in the quality of cardiopulmonary resuscitation (CPR) according to driving patterns encountered during ambulance transport, using a virtual reality simulator.

Design Prospective, cross-over, randomised study.

Setting This study was conducted at the National Fire Service Academy, Cheonan-si, Korea.

Participants Emergency medical technicians (39 men and 9 women) attending the National Fire Service Academy for clinical training with $\geq 6$ months field experience or having performed $\geq 10 \mathrm{CPR}$. Individuals who withdrew consent were excluded.

Outcome measures CPR quality parameters (eg, chest compression depth and its variability).

Results Chest compressions were performed for $8 \mathrm{~min}$ each in a stationary and driving state. The mean chest compression depths were $54.8 \mathrm{~mm}$ and $55.3 \mathrm{~mm}$ during these two states, respectively $(p=0.41)$. The SD of the chest compression depth was significantly higher while in the driving $(7.6 \mathrm{~mm})$ than in the stationary state $(6.5 \mathrm{~mm}$; $\mathrm{p}=0.04)$. The compression depths in the speed bump and sudden stop sections were $51.5 \mathrm{~mm}$ and $50.6 \mathrm{~mm}$, respectively, which was shallower than those in all other sections $(p<0.001)$. The correct hand position rate was low in the speed bump, sudden stop and right-hand cornering sections $(65.4 \%, 71.5 \%$ and $72.5 \%$, respectively; $\mathrm{p}=0.001$ )

Conclusions Although we found no differences in chest compression quality parameters between the stationary and driving states, the variability in the chest compression depth increased in the driving state. When comparing CPR quality parameters according to driving patterns, we noted a shallower compression depth, increased variability and decreased correct hand position rate in the speed bump, sudden stop and right-hand cornering sections. The clinical significance of these changes in CPR quality during ambulance transport remains to be determined. Future studies on how to reduce changes in the quality of CPR (including research on equipment development) are needed.

\section{Strengths and limitations of this study}

- This is the first study to evaluate changes in cardiopulmonary resuscitation (CPR) quality according to various driving patterns (ie, acceleration, deceleration, turning, cornering, speed bumps and sudden stops).

- A virtual reality ambulance simulator and driving system that were developed to safely conduct first aid training inside an ambulance were used to achieve constant driving patterns throughout the trials, which is difficult during real ambulance transport.

- The results obtained with the virtual reality ambulance simulator may differ from those observed in real-life situations.

- As some driving pattern sections (eg, the speed bump as well as left and right turn sections) appeared for only a few seconds, some CPR quality indicators, such as chest compression rate, correct recoil rate and total time taken to change the chest compressor, could not be assessed.

\section{BACKGROUND}

The average time from the reporting of a cardiac arrest event to the arrival on the scene by emergency medical services (EMS) is approximately $9 \mathrm{~min}$, and it takes approximately 20 min to reach the hospital thereafter. ${ }^{1}$ The duration of cardiopulmonary resuscitation (CPR) in a patient with an out-of-hospital cardiac arrest (OHCA) by EMS accounts for over $50 \%$ of the prehospital time, and much of this time is spent performing CPR during the ambulance transport. However, the quality of CPR performed during ambulance transport has been reported to not meet the criteria proposed in the American 
Heart Association and European Resuscitation Council guidelines. ${ }^{2}{ }^{3}$ CPR performed during ambulance transport has a hands-off ratio of 0.27 , which is higher than the on-scene ratio of 0.19 . Moreover, although the chest compression depth during ambulance transport varies across studies, some studies have reported that it is lower than the depth for on-scene CPR or that performed while stationary. ${ }^{4-7}$

Whereas previous studies have compared the quality of CPR performed during ambulance transport and that performed on scene or in a stationary state, no study has evaluated changes in CPR quality according to various driving patterns (ie, acceleration, deceleration, turning, cornering, speed bumps and sudden stops). When emergency medical technicians (EMTs) perform CPR inside an ambulance, they cannot wear a seat belt and cannot rely on any devices to stabilise their position. Conducting studies on CPR performed in an ambulance operating in real-time presents a high risk of injury to those involved as well as ethical issues. ${ }^{89}$ Therefore, we analysed the changes in the quality of CPR parameters according to various driving patterns encountered during actual patient transport in a safe environment, using a virtual reality (VR) ambulance simulator and driving system.

\section{METHODS}

\section{Patient involvement}

No patients were involved in this study.

\section{Study design and population}

We performed a prospective, cross-over, randomised study to measure changes in CPR quality parameters according to driving patterns encountered during ambulance transport using a VR ambulance simulator. We recruited volunteers after explaining the study to paramedics who entered the National Fire Service Academy for EMS clinical training. The study population comprised 48 EMTs or nurses who had $\geq 6$ months of field experience or had performed $\geq 10 \mathrm{CPR}$. Individuals who initially consented to participate but subsequently withdrew their consent were excluded.

\section{VR ambulance simulator and driving system}

We used a VR ambulance simulator that was developed to safely conduct first aid training inside an ambulance. The simulator comprised an ambulance, moving platform and a VR driving system. The simulation ambulance was placed on top of a moving platform with six axes, and the movement of the ambulance, which was controlled by the VR driving system connected to the ambulance, allowed the participants to experience an environment similar to that inside an ambulance operating in real time (figure 1A). ${ }^{10}$

To develop the VR driving system, we selected 10 driving patterns that may be encountered during actual driving: section 1, stationary; section 2, acceleration; section 3, constant velocity; section 4, right-hand cornering; section 5 , left-hand cornering; section 6 , deceleration; section 7 ,

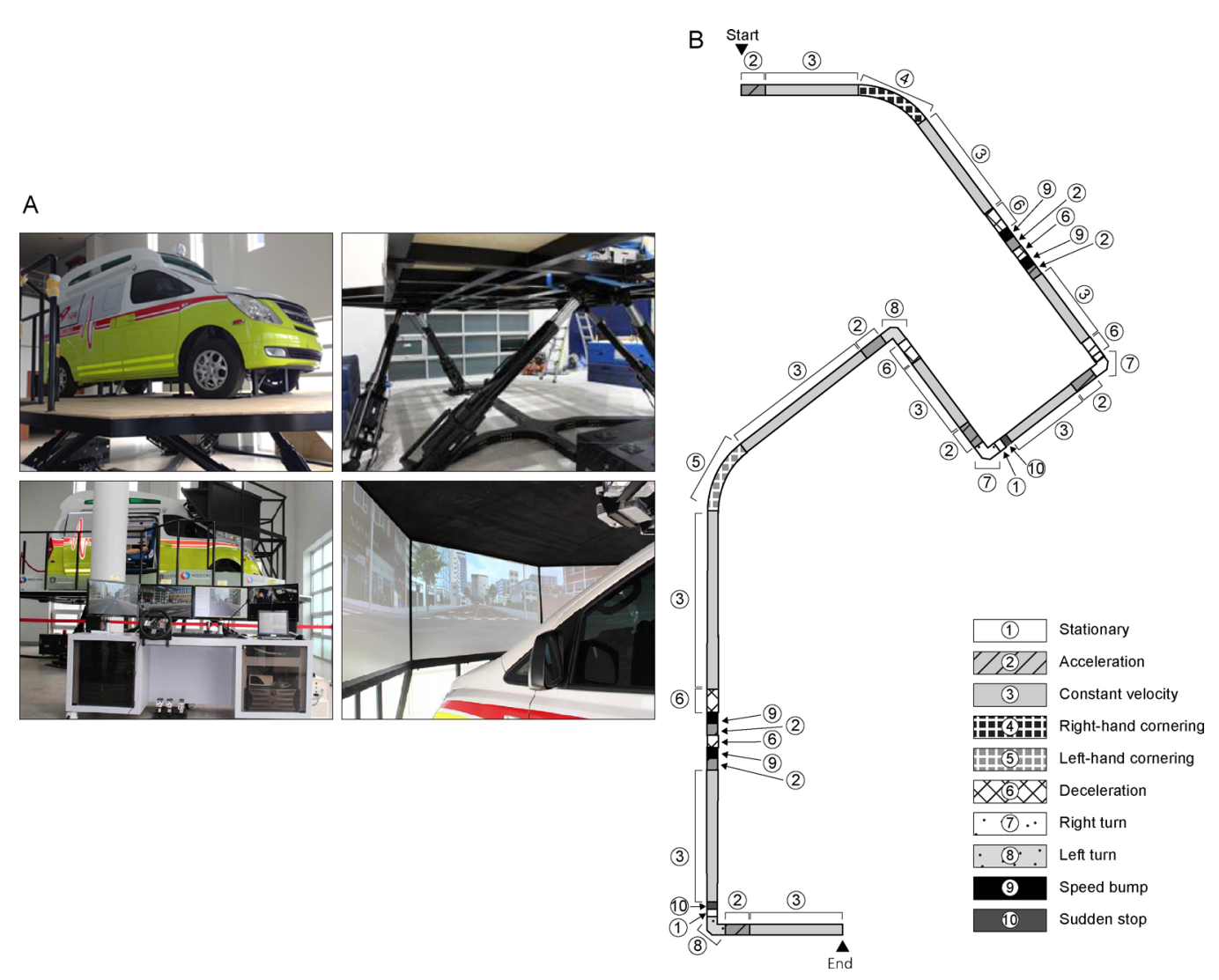

Figure 1 Virtual reality ambulance simulator $(A)$ a virtual reality driving route $(B)$. 
right turn; section 8, left turn; section 9, speed bump and section 10, sudden stop. Based on routes from areas in close proximity to our emergency centre, we developed $6739 \mathrm{~m}$ of VR driving system, including the 10 driving patterns mentioned above (figure $1 \mathrm{~B}$ ). The maximum virtual driving speed was set at $60 \mathrm{~km} /$ hour, considering the city's speed limit. Moreover, it was set at $60 \mathrm{~km} /$ hour for the constant velocity and cornering sections and $20 \mathrm{~km} /$ hour for the right turn, left turn and speed bump sections. The sudden stop section was defined as travelling at a constant velocity of $60 \mathrm{~km} /$ hour and then coming to a sudden stop at a signal. The right-hand and left-hand cornering sections were designed to have an arc with a radius of $1000 \mathrm{~m}$, while the right and left turn sections were designed to have an arc with a radius of $10 \mathrm{~m}$.

\section{Study protocol}

The participants were paired and performed two trials of chest compression-only CPR for $8 \mathrm{~min}$ each in the stationary state and under virtual driving conditions on a Rodam CPR Manikin (BT-CPEA, BT, Goyang-si, Korea) that was placed on top of a bed inside the ambulance simulator. During the trial, one participant performed chest compressions while the other participant waited beside the patient's head; the participants alternated every $2 \mathrm{~min}$, according to the researcher's instructions. The second trial was performed after a 1-hour resting period to minimise rescuer fatigue. To avoid potential bias, no feedback was given to participants after each trial.

The order in which the paired participants performed CPR in any state (stationary or driving) and the order in which they performed chest compressions was randomly assigned using permuted four-block randomisation.

\section{Measures}

CPR quality parameters were collected and analysed using the PC Skill Reporting System (BT-CPEA, BT). The primary endpoints were the mean chest compression depth and the variability in compression depth. Additional endpoints included the chest compression rate, correct compression depth rate, correct recoil rate, correct hand position rate and total time taken to change the chest compressor every $2 \mathrm{~min}$. The correct compression depth rate was defined as the ratio of the number of compressions to a depth of at least $50 \mathrm{~mm}$ to the total number of compressions performed. The correct recoil rate was defined as the ratio of the number of compressions allowed to release to within $5 \mathrm{~mm}$ of the original chest height to the total number of compressions performed. The correct hand position rate was defined as the ratio of the number of compressions performed over the lower half of the sternum to the total number of compressions performed.

After completing two practice sessions, the participants were asked to compare the differences between real-life driving conditions and the simulation situation on a $100 \mathrm{~mm}$ Visual Analogue Scale (VAS), with 0 representing 'not similar at all' and 100 representing 'same'.

\section{Statistical analysis}

The sample size was calculated based on the mean compression depth, considering the cross-over design. A difference of $5 \mathrm{~mm}$ in the mean compression depth between the stationary and driving states was selected as the minimum clinically significant value. We considered the SD of the difference between the two states to be 8.4. The SD of the difference was calculated assuming that the SD of each group was 10.9 and that the correlation coefficient between the two states was $0.7 .{ }^{11}$ We estimated that a sample size of 24 pairs (48 participants) would be sufficient to evaluate the primary outcome at a significance level of 0.05 (two sided) with $80 \%$ power.

Discrete variables are presented as rates $(\%)$ and continuous variables as means $\pm \mathrm{SD}$ or means ( $95 \% \mathrm{CIs})$. We used the independent t-test for the comparison of continuous variables and the Fisher's exact test to analyse categorical variables. A linear mixed model was used to assess the differences in CPR quality parameters considering the cross-over study design. Three fixed effects were included in the model: one between-subject effect (the sequence effect; first, CPR is performed in the stationary state, then in the driving state and vice versa); two within-subject effects (the group effect; CPR in the stationary and driving states) and the period effect (first, second). To confirm the variability in the chest compression quality, the $\mathrm{SD}$ and coefficients of variation $(\mathrm{SD} /$ mean) were also analysed. A $\mathrm{p}<0.05$ was considered statistically significant. PASS (V.12, NCSS, LLC, Kaysville, Utah, USA) and SAS V.9.2 (SAS Institute) were used for the statistical analyses of the collected data.

\section{RESULTS}

The study population comprised 48 EMTs (39 men, 81.3\%) enrolled in the National Fire Service Academy for EMS clinical training, with a mean age of $31.9 \pm 3.8$ years. All registered participants were certified level 1 EMT (equivalent to EMT-intermediate in the North American EMS) or nurses. The demographic characteristics of the participants are summarised in table 1. Except for the length of the paramedic career, there were no significant differences between the demographics of the stationary and driving trial first groups.

The mean chest compression depths in the stationary and driving states were $54.8 \mathrm{~mm}$ and $55.3 \mathrm{~mm}$, respectively; no significant difference was observed between the two states $(\mathrm{p}=0.41$; table 2$)$. The chest compression rates in the stationary and driving states were 107.2 per min and 106.2 per min, respectively; again, no significant difference was observed $(\mathrm{p}=0.61$; table 2$)$. Furthermore, we found no significant differences in the correct compression depth rate, correct recoil rate, correct hand position rate and total time taken to change the chest compressor between the stationary and driving states (table 2). However, the SD of the chest compression depth in the driving state of $7.6 \mathrm{~mm}$ was significantly higher than that of $6.5 \mathrm{~mm}$ observed in the stationary state $(\mathrm{p}=0.04)$. 


\begin{tabular}{|c|c|c|c|}
\hline Variable & $\begin{array}{l}\text { Stationary } \\
\text { trial first } \\
\text { group }\end{array}$ & $\begin{array}{l}\text { Driving } \\
\text { trial first } \\
\text { group }\end{array}$ & $P$ values \\
\hline Age (years), mean $\pm S D$ & $32.5 \pm 4.3$ & $31.5 \pm 3.4$ & 0.37 \\
\hline \multicolumn{4}{|l|}{ Sex, N (\%) } \\
\hline Male & $19(79.1)$ & 20 (83.3) & 0.99 \\
\hline Female & $5(20.9)$ & $4(16.7)$ & \\
\hline Height (cm), mean \pm SD & $172.7 \pm 8.0$ & $172.8 \pm 6.0$ & 0.98 \\
\hline Weight $(\mathrm{kg})$, mean $\pm S D$ & $70.9 \pm 13.7$ & $66.5 \pm 6.4$ & 0.16 \\
\hline $\begin{array}{l}\text { Duration of paramedic } \\
\text { career (months), } \\
\text { mean } \pm S D\end{array}$ & $70 \pm 33$ & $46 \pm 21$ & 0.01 \\
\hline Occupation, N (\%) & & & 0.52 \\
\hline Level $1 \mathrm{EMT}^{*}$ & $19(79.1)$ & $16(66.6)$ & \\
\hline Nurse & $5(20.9)$ & $8(33.4)$ & \\
\hline
\end{tabular}

${ }^{*} \mathrm{~A}$ level $1 \mathrm{EMT}$ is equivalent to an EMT-intermediate in the North American emergency medical system.

EMT, emergency medical technician.

In the comparison of CPR quality parameters according to driving patterns, the mean chest compression depths in the speed bump and sudden stop sections were $51.5 \mathrm{~mm}$ and $50.6 \mathrm{~mm}$, respectively, which was lower than those in all other sections $(p<0.001$; table 3$)$. The correct compression depth rates in the speed bump, sudden stop and right-hand cornering sections were $66.5 \%, 66.6 \%$, and $67.8 \%$, respectively; these rates were lower than those in all other sections, but the differences were not statistically significant $(p=0.15)$. The correct hand position rates in the speed bump, sudden stop and right-hand cornering sections were $65.4 \%, 71.5 \%$ and $72.5 \%$, respectively, which was significantly lower than those in all other sections $(p=0.001$; table 3 ). The variability analysis of the compression depth by sections according to driving patterns showed that the SD and coefficient of variation in the speed bump and sudden stop sections were significantly higher than those in the other sections $(p<0.001$ for both; figure 2).
With respect to the participants' responses on how realistic the virtual driving programme was, the mean VAS score was $70.1 \pm 14.7 \mathrm{~mm}$. The mean VAS score reported by the EMTs was similar to that reported by the nurses $(73 \pm 15.8 \mathrm{~mm}$ vs $65 \pm 9.4 \mathrm{~mm}, \mathrm{p}=0.09)$.

\section{DISCUSSION}

Providing high-quality CPR to patients with cardiac arrest in and out of the hospital is crucial for improving patient survival and achieving good neurological prognoses. ${ }^{12-15}$ It is important to improve CPR quality as CPR during ambulance transport accounts for a major portion of OHCA care. However, few studies have investigated the quality of CPR performed during ambulance transport, and none have assessed the quality of CPR parameters according to driving patterns to date. In this study, we found no significant differences in the mean CPR quality parameters (such as the chest compression depth and rate) between the stationary and driving states. However, the SD of the chest compression depth showed a difference between the two states. Similar results were observed in a previous study. Roosa et al compared on-scene and ambulance transport CPR and found that the chest compression depths were 1.74 inch and 1.68 inch and the chest compression rates 109 per min and 106 per min in the stationary and driving states, respectively; no significant differences were observed between the two states. However, the compression depth variability values (inch) in the stationary and driving states were 0.20 and 0.26 , respectively, indicating a significant difference $(\mathrm{p}<0.01){ }^{11}$ This is consistent with the findings of our study, in which a higher chest compression depth SD was observed during VR transport.

When we compared CPR quality parameters between the different sections during the driving state, the chest compression depth was lower in the speed bump and sudden stop sections, and the variability in the chest compression depth increased. These results confirm that the chest compression depth in these sections was too low or too deep, indicating that, although the mean chest compression depth exceeded $50 \mathrm{~mm}$ in these sections, the

Table 2 Comparison of cardiopulmonary resuscitation quality between the stationary and driving state

\begin{tabular}{|c|c|c|c|c|c|}
\hline \multirow[b]{2}{*}{ Variable } & \multirow[b]{2}{*}{ Stationary state } & \multirow[b]{2}{*}{ Driving state } & \multicolumn{3}{|l|}{$P$ values } \\
\hline & & & Sequence & Period & Group \\
\hline Chest compression depth (mm) & 54.8 (51.9 to 57.6$)$ & $55.3(52.4$ to 58.1$)$ & 0.48 & 0.26 & 0.41 \\
\hline Correct hand position rate (\%) & 83.1 (75.4 to 90.8$)$ & 80.0 (72.4 to 87.7$)$ & 0.05 & 0.59 & 0.25 \\
\hline Correct compression depth rate (\%) & 71.1 (58.6 to 83.7 ) & 74.2 (61.7 to 86.8$)$ & 0.19 & 0.26 & 0.46 \\
\hline
\end{tabular}

Except for the $\mathrm{p}$ values, data are shown as means adjusted for randomisation sequences and time periods and their $95 \%$ Cls, assessed by a linear mixed model. 


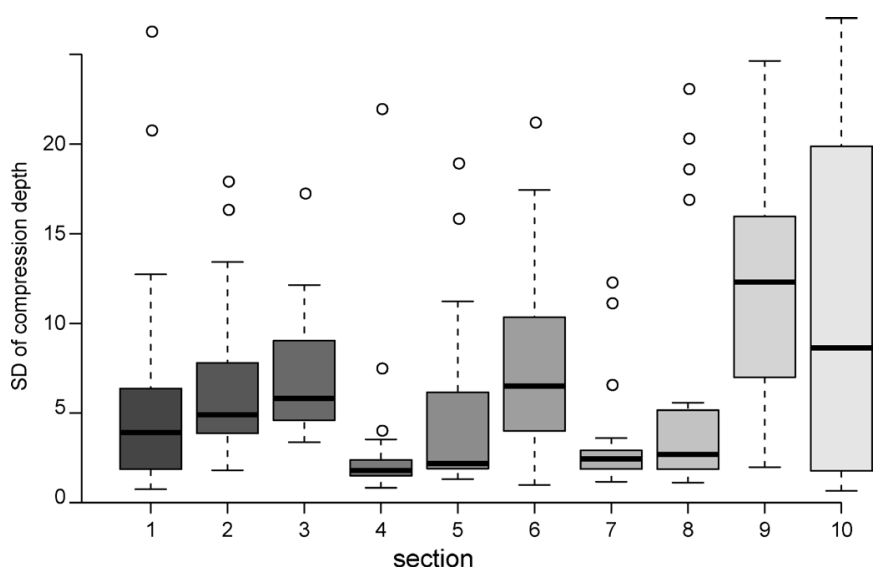

Figure 2 Box plot demonstrating the SD of the compression depth according to the driving pattern. The upper horizontal line of the box indicates the 75th percentile, the lower horizontal line the 25th percentile, the horizontal line within the box the median, the upper horizontal bar outside the box the maximum value excluding outliers and the lower horizontal bar outside the box the minimum value excluding outliers. Circles represent outliers $\left(<25\right.$ th percentile $-1.5^{\star}$ IQR or $>75$ th percentile $+1.5^{\star} \mathrm{IQR}$ ).

chest compressions may not have been performed properly, or excessive compression may have caused additional chest injuries. Our results also showed that the correct hand position rate decreased in both aforementioned sections; this may have resulted from the sudden shaking of the body in those sections. It is highly likely that such results may be found under city driving conditions in which the ambulance may be travelling at a high speed in an emergency situation but may need to come to a sudden stop due to the presence of speed bumps or heavy traffic. Therefore, when studying CPR quality during patient transport, the road conditions, flow of traffic and driving behaviour of the driver should be considered.

In the right-hand cornering section, the correct hand position rate decreased, which may be attributed to the influence of the centrifugal force generated as the vehicle turned. When a vehicle performs right-hand cornering, the EMT inside the vehicle is subjected to a centrifugal force to the left in relation to the vehicle being driven. In this study, when the patient lay in the ambulance driving direction and the EMT performed chest compressions while positioned to the left of the patient, the EMT's centre of mass (CoM) leaned forward. In such situations, the centrifugal force is added to the front of the EMT as the vehicle turns to the right; this may cause the EMT to easily lose balance instantaneously. On the other hand, when performing left-hand cornering, the centrifugal force exerted is directed towards the back of the EMT performing chest compressions. Since the centrifugal force is applied in the direction opposite to the forward-leaning CoM, the forces offset each other and relatively less shaking is experienced when compared with performing right-hand cornering. However, during sharp cornering, the centrifugal force directed towards the back may be greater than the force of the CoM, which 
may produce a different outcome. Studies that used stabilisation devices to identify improvements in the efficiency of CPR during ambulance transport showed that the effective chest compression rate improved and the no-flow fraction decreased to a greater extent when a device with $69 \mathrm{~cm}$ high bars installed in the front and both sides of the EMT was used with no other safety devices in the back, than when a stabilisation device was used that covered all sides. ${ }^{16}$ This result showing that high-quality CPR was achieved without any safety devices in the back of the EMT, indirectly supports the findings of our study.

Chung et al compared the quality of chest compressions under four different conditions: stationary, $30 \mathrm{~km} /$ hour, $60 \mathrm{~km} /$ hour and $90 \mathrm{~km} /$ hour. They reported that chest compressions with an adequate depth decreased and those with an excessive depth increased as the vehicle's speed increased. ${ }^{17}$ However, in their study, CPR was performed when the vehicle reached the target speed; thus, they only compared the results under a constant velocity state and did not examine CPR quality during acceleration or deceleration states. When designing the VR driving system for our study, we established a constant velocity $(60 \mathrm{~km} /$ hour $)$ as well as acceleration and deceleration sections to examine changes in the quality of CPR parameters according to changes in velocity. However, our findings did not show any differences in CPR quality between the constant velocity, acceleration and deceleration sections. This may be attributed to the fact that we limited the maximum velocity of the VR ambulance to $60 \mathrm{~km} /$ hour (the speed limit of the city where this study was performed) and set it to drive with sufficient distance to avoid rapid acceleration and deceleration, considering the safety of the EMT. Second, the VR driving system may not have been similar enough to real-life driving conditions, as evidenced by the participants' mean VAS score.

Although our findings point to a shallower chest compression depth, increased variability in chest compression depth and lower correct hand position rate during certain sections, we cannot conclude that high-quality CPR was not performed during ambulance transport. We could not assess some CPR quality indicators such as the mean chest compression rate and chest compression fraction. Moreover, although the chest compression depth was shallower in the speed bump and sudden stop sections than in the other sections, the mean compression depth in these sections was $>50 \mathrm{~mm}$, and the correct compression depth rate was similar to that of other driving sections.

The use of a mechanical chest compression device may compensate for the poor quality of CPR during specific driving patterns. The International Liaison Committee on Resuscitation published a weak recommendation that mechanical chest compression devices should be considered in particular settings in which high-quality manual CPR may be impractical or dangerous to rescuers, such as in a moving ambulance. ${ }^{18}$ However, large randomised controlled trials and meta-analyses do not support the notion that such devices are superior to manual CPR despite them providing consistent and high-quality chest compressions. ${ }^{19-22}$ Moreover, an analysis of the cost-effectiveness of the use of mechanical compression devices in OHCA showed that they were more costly and less effective than manual chest compressions. ${ }^{23}$ Therefore, further studies are needed to evaluate the routine use of mechanical devices in OHCA.

Our study has some limitations. First, this was a manikin study that used a VR driving programme; therefore, the results may vary from those observed in real-life situations. The VAS in terms of how realistic the VR driving programme was, as evaluated by the participants, was $70 \mathrm{~mm}$. When asked about how the VR driving programme was different from real-life driving conditions, the participants explained that they often drive at speeds higher than $60 \mathrm{~km} /$ hour in real-life situations and that their body moves more violently while performing CPR due to sudden stops and more sudden acceleration and deceleration. However, the VR driving system used in this study was designed to prioritise the safety of the participants over an accurate reproduction of real-life situations. Second, our study required CPR to be performed for $8 \mathrm{~min}$. This setting was based on the actual time required for an ambulance to reach the hospital. ${ }^{5425}$ However, in the case of rural prehospital systems, a decline in the quality of CPR due to a longer transport time may occur. Third, in our study, continuous chest compressions without rescue breathing were performed. In our virtual driving system, some driving patterns (eg, the speed bump, sudden stop, right turn or left turn sections) occurred within a few seconds or less. If the participants would have performed conventional CPR, including rescue breathing, they may have switched between rescue breathing and chest compressions or may have performed two rescue breathing trials during such a short driving pattern. In these cases, we would not have been able to obtain the quality indicators of the chest compressions. However, as we chose the continuous chest compression method for this study, we could not evaluate the effect of the driving pattern on rescue breathing or rescuers switching. Moreover, some quality indicators, such as the chest compression rate, correct recoil rate and total time taken to change the chest compressor, could not be assessed because of the short duration of some driving patterns. Finally, the participants recognised that they were being evaluated and monitored. This may have caused a Hawthorne effect, meaning that they may have performed better than they would during real-life ambulance transport; this may explain our finding that the difference of the mean compression depth between the two states was only $0.5 \mathrm{~mm}$. The difference between real-life driving conditions and the VR simulation could have also been the reason for this small difference.

\section{CONCLUSIONS}

We observed no differences in chest compression quality parameters between the stationary and driving states but 
noted an increase in the variability of the chest compression depth. Moreover, the compression depth was shallower, its variability higher, and the correct hand position rate lower in the speed bump and sudden stop sections when compared with all other sections. In the right-hand cornering section, only the correct hand position rate decreased. The clinical significance of these changes in CPR quality during ambulance transport remains to be determined. Future studies on how to reduce changes in the quality of CPR (including research on equipment development) are needed.

Acknowledgements We would like to thank the instructors of the National Fire Service Academy for data collection. We would also like to thank Dong-Su Jang (medical illustrator) for his help with the illustrations.

Contributors YSP, together with JHB, DMS and HSC designed this study. JHB, MJK, JSY and JHK contributed to data acquisition. HSL and YSP performed the data analysis. MJK, JSY and JHK helped interpret the experimental results. YSP and JHB drafted this manuscript. All authors read and approved the final manuscript.

Funding This study was supported by a faculty research grant of the Yonsei University College of Medicine (no. 6-2015-0080).

Disclaimer The funding body had no role in the design of the study; the collection, analysis and interpretation of data and in writing the manuscript.

Competing interests None declared.

Patient consent Not required.

Ethics approval This study was approved by the Institutional Review Board of the Yonsei University College of Medicine, Severance Hospital (no. 4-2015-0564).

Provenance and peer review Not commissioned; externally peer reviewed.

Data sharing statement The datasets used and/or analysed during the current study are available from the corresponding author on reasonable request.

Open access This is an open access article distributed in accordance with the Creative Commons Attribution Non Commercial (CC BY-NC 4.0) license, which permits others to distribute, remix, adapt, build upon this work non-commercially, and license their derivative works on different terms, provided the original work is properly cited, appropriate credit is given, any changes made indicated, and the use is non-commercial. See: http://creativecommons.org/licenses/by-nc/4.0/.

\section{REFERENCES}

1. Sladjana A, Gordana P, Ana S. Emergency response time after outof-hospital cardiac arrest. Eur J Intern Med 2011;22:386-93.

2. Hazinski MF, Nolan JP, Aickin R, et al. Part 1: Executive summary: 2015 international consensus on cardiopulmonary resuscitation and emergency cardiovascular care science with treatment recommendations. Circulation 2015;132:S2-39.

3. Monsieurs KG, Nolan JP, Bossaert LL, et al. European resuscitation council guidelines for resuscitation 2015: Section 1. Executive summary. Resuscitation 2015;95:1-80.

4. Olasveengen TM, Wik L, Steen PA. Quality of cardiopulmonary resuscitation before and during transport in out-of-hospital cardiac arrest. Resuscitation 2008;76:185-90.

5. Ødegaard S, Olasveengen T, Steen PA, et al. The effect of transport on quality of cardiopulmonary resuscitation in out-of-hospital cardiac arrest. Resuscitation 2009;80:843-8.
6. Havel C, Schreiber W, Riedmuller E, et al. Quality of closed chest compression in ambulance vehicles, flying helicopters and at the scene. Resuscitation 2007;73:264-70.

7. Wang HC, Chiang WC, Chen SY, et al. Video-recording and timemotion analyses of manual versus mechanical cardiopulmonary resuscitation during ambulance transport. Resuscitation 2007;74:453-60.

8. Slattery DE, Silver A. The hazards of providing care in emergency vehicles: an opportunity for reform. Prehosp Emerg Care 2009;13:388-97.

9. Ray AF, Kupas DF. Comparison of crashes involving ambulances with those of similar-sized vehicles. Prehosp Emerg Care 2005;9:412-5.

10. Shin DM. Development of VR based simulated ambulance for training paramedics, 2015. National Digital Science Library http:// www.ndsl.kr/ndsl/commons/util/ndslOriginalView.do?dbt=TRKO\& $\mathrm{cn}=$ TRKO201600011222\&rn=\&url=\&pageCode=PG18 (accessed 12 Jul 2018).

11. Roosa JR, Vadeboncoeur TF, Dommer PB, et al. CPR variability during ground ambulance transport of patients in cardiac arrest. Resuscitation 2013;84:592-5.

12. Berg RA, Hemphill R, Abella BS, et al. Part 5: adult basic life support: 2010 American Heart Association Guidelines for Cardiopulmonary Resuscitation and Emergency Cardiovascular Care. Circulation 2010;122:S685-S705.

13. Herlitz J, Svensson L, Holmberg S, et al. Efficacy of bystander CPR: intervention by lay people and by health care professionals. Resuscitation 2005;66:291-5.

14. Bobrow BJ, Clark LL, Ewy GA, et al. Minimally interrupted cardiac resuscitation by emergency medical services for out-of-hospital cardiac arrest. JAMA 2008;299:1158-65.

15. Koster RW, Baubin MA, Bossaert LL, et al. European resuscitation council guidelines for resuscitation 2010 section 2 . Adult basic life support and use of automated external defibrillators. Resuscitation 2010;81:1277-92.

16. Foo NP, Chang JH, Su SB, et al. A stabilization device that promotes the efficiency of cardiopulmonary resuscitation during ambulance transportation to the level as under non-moving conditions. PLOS One 2014;9:e107960.

17. Chung TN, Kim SW, Cho YS, et al. Effect of vehicle speed on the quality of closed-chest compression during ambulance transport. Resuscitation 2010;81:841-7.

18. Soar J, Callaway CW, Aibiki M, et al. Part 4: Advanced life support: 2015 international consensus on cardiopulmonary resuscitation and emergency cardiovascular care science with treatment recommendations. Resuscitation 2015;95:e71-e120.

19. Wik L, Olsen JA, Persse D, et al. Manual vs. integrated automatic load-distributing band CPR with equal survival after out of hospital cardiac arrest. The randomized CIRC trial. Resuscitation 2014;85:741-8.

20. Rubertsson S, Lindgren E, Smekal D, et al. Mechanical chest compressions and simultaneous defibrillation vs conventional cardiopulmonary resuscitation in out-of-hospital cardiac arrest: the LINC randomized trial. JAMA 2014;311:53-61.

21. Perkins GD, Lall R, Quinn T, et al. Mechanical versus manual chest compression for out-of-hospital cardiac arrest (PARAMEDIC): a pragmatic, cluster randomised controlled trial. Lancet 2015;385:947-55.

22. Gates S, Quinn T, Deakin CD, et al. Mechanical chest compression for out of hospital cardiac arrest: Systematic review and metaanalysis. Resuscitation 2015;94:91-7.

23. Marti J, Hulme C, Ferreira Z, et al. The cost-effectiveness of a mechanical compression device in out-of-hospital cardiac arrest. Resuscitation 2017;117:1-7.

24. Cheskes S, Byers A, Zhan C, et al. CPR quality during out-of-hospital cardiac arrest transport. Resuscitation 2017;114:34-9.

25. Kurz MC, Dante SA, Puckett BJ. Estimating the impact of offbalancing forces upon cardiopulmonary resuscitation during ambulance transport. Resuscitation 2012;83:1085-9. 